Ana Vrgoč

Zvonimir Tomičević $\bowtie$

Andrija Zaplatić

François Hild

https://doi.org/10.21278/TOF.452024020

ISSN 1333-1124

eISSN 1849-1391

\title{
DAMAGE ANALYSIS IN GLASS FIBRE REINFORCED EPOXY RESIN VIA DIGITAL IMAGE CORRELATION
}

\begin{abstract}
Summary
The objective of the presented experimental study was to perform monotonic tensile tests and observe the damage occurring on the surface of a glass fibre mat reinforced epoxy resin. The experiments were performed on dogbone specimens machined from a $5.8 \mathrm{~mm}$ thick composite plate. The macroscopic response was obtained from the load data and 2D digital image correlation. The finite element-based Digital Image Correlation was employed to measure displacement fields and calculate strain fields across the entire region of interest. In addition, the grey level residuals extracted from the correlation analyses were utilized to detect discontinuities (i.e. damage initiation and growth) on the surface of the investigated specimens.
\end{abstract}

Key words: $\quad$ Damage, Digital Image Correlation, registration residuals, glass fibre composite

\section{Introduction}

Due to their high stiffness-to-weight ratio and their unique ability to tailor material properties to meet specific design requirements, fibre-reinforced polymer (FRP) composites are in constant, ever-increasing use in critical-safety and lightweight structures. In addition to enhanced resistance to environmental conditions and fatigue, FRPs exhibit good formability during manufacturing and are easy to maintain [1]-[3]. Various industry branches (especially automotive, aeronautics and aerospace) aim to exploit more and more the potential of FRPs. However, the full understanding of their behaviour in the service life remains a challenge and calls for advanced material characterization methods. In particular, the identification of material properties and the observation of damage mechanisms via non-destructive methods have become crucial for an accurate prediction of structural responses up to and beyond the inception of damage and fracture.

In recent years, Digital Image Correlation (DIC) has proven to be an accurate and versatile tool for bridging the gap between experimental tests and mechanical models [4],[5]. This contactless, full-field measurement technique has been widely employed in experimental solid mechanics to extract meaningful information from tests [6]. Due to the increased use of composite materials, DIC has been utilized to identify material parameters and investigate damage mechanisms occurring within such complex and heterogeneous materials [7]-[9]. 
The research conducted herein focuses on the observation of damage initiation and growth in a glass fibre reinforced epoxy resin composite during monotonic tensile tests. Specimens were cut from a composite plate at different angles to estimate their global response. In the present case, a regularized FE-based DIC was utilized to measure displacement fields and calculate strain fields on the surface of tested specimens. Furthermore, grey level residual maps extracted from correlation analyses were studied to detect the damage initiation and to monitor the subsequent crack growth.

\section{Experimental study}

In this section, the experimental protocol employed in the present study is discussed. First, the properties of the investigated composite material are presented. The dogbone specimens were cut from a composite plate along three different directions to estimate the macroscopic response and its dependence on the angular sampling. Experimental data were the applied force measured by the load cell of a testing machine and a series of images of specimen surfaces acquired during each of three experiments. A brief overview of the global DIC algorithm is given, followed by a summary of hardware parameters employed in the correlation procedure.

\subsection{Fibre-reinforced polymer (FRP) composite}

Experiments were performed on a continuous glass fibre mat reinforced epoxy resin composite. The manufacturing process of the material consisted of manual lay-up followed by compression moulding. The laminate consisting of 12 layers of R-glass fibre mat placed in $438 \mathrm{~g}$ of epoxy resin mixture (LG 285 laminating resin, GRM Systems, Germany) was cured under pressure for $24 \mathrm{~h}$ at room temperature. Subsequently, post-curing was performed at $100{ }^{\circ} \mathrm{C}$ for $3 \mathrm{~h}$. Micrographs of the investigated material are presented in Fig. 1. Since vacuum was not applied during moulding after lay-up, the micrographs shown below reveal the presence of air bubbles (i.e. voids).

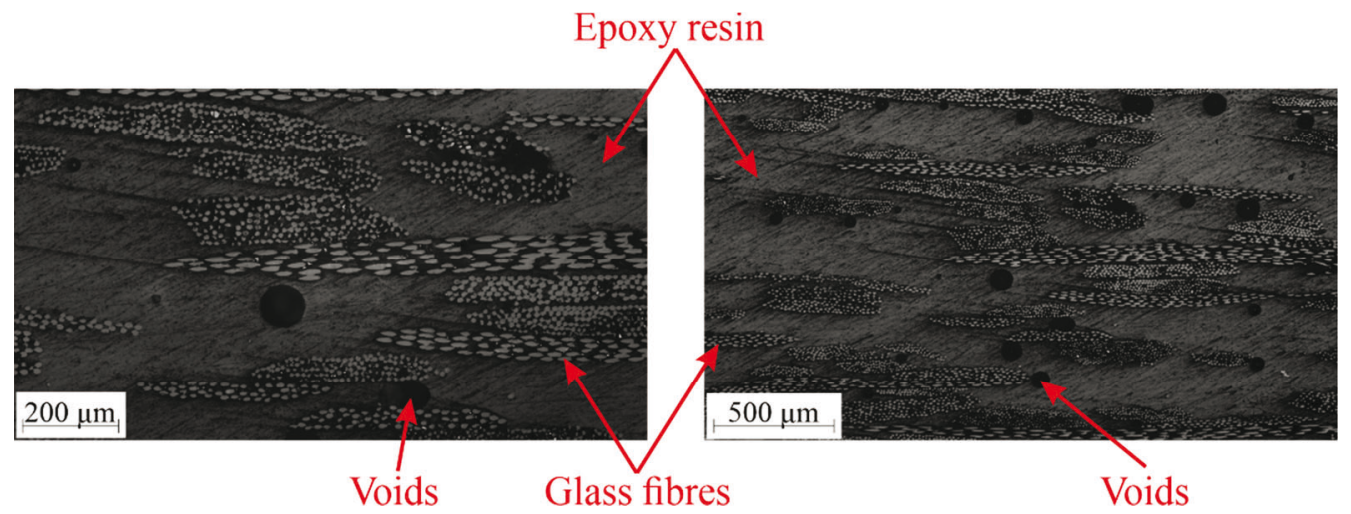

Fig. 1 Micrographs of composite cross section at two different magnifications.

\subsection{Digital Image Correlation (DIC)}

Digital Image Correlation is one of the most commonly used techniques for performing full-field measurements based on registering the grey level images of specimen surfaces Error! Reference source not found. The images acquired during the experiment are correlated to measure displacement fields over the entire Region of Interest (ROI) assuming the conservation of grey levels at any pixel location $\mathbf{x}$ between the reference image, $f(\mathbf{x})$, usually taken in the undeformed state, and the deformed image, $g(\mathbf{x})$ :

$$
f(\mathbf{x})=g(\mathbf{x}+\mathbf{u}(\mathbf{x}))
$$


where $\mathbf{u}(\mathbf{x})$ is the sought displacement field. The DIC technique consists of minimising the sum of square differences $\Phi_{c}^{2}$ over the ROI with respect to the sought displacement field [10]:

$$
\Phi_{c}^{2}=\int_{\text {ROI }} \varphi_{c}^{2}(\mathbf{x}) \mathrm{d} \mathbf{x}
$$

where $\varphi_{c}(\mathbf{x})$ defines the field of correlation residuals field:

$$
\varphi_{C}(\mathbf{x})=f(\mathbf{x})-g(\mathbf{x}+\mathbf{u}(\mathbf{x})) .
$$

Compared to local approaches, which analyse the series of subsets independently, the global DIC approach applied herein assumes the continuity of displacement fields over the whole ROI. Since the presented minimisation is a nonlinear and ill-posed problem, the displacement field is written as:

$$
\mathbf{u}(\mathbf{x})=\sum_{n} u_{n} \Psi_{n}(\mathbf{x})
$$

where $u_{\mathrm{n}}$ are the nodal displacements associated with the selected shape functions $\psi_{n}$. If small increments are assumed, a Taylor expansion is applied:

$$
g(\mathbf{x}+\mathbf{u}(\mathbf{x})+\delta \mathbf{u}(\mathbf{x})) \approx g(\mathbf{x}+\mathbf{u}(\mathbf{x}))+\delta \mathbf{u}(\mathbf{x})) \cdot \nabla f(\mathbf{x}) .
$$

The Gauss-Newton iterative algorithm is utilized to solve linear systems:

$$
[\mathbf{M}]\{\delta \mathbf{u}\}=\left\{\mathbf{b}^{i}\right\},
$$

where $[\mathbf{M}]$ is the DIC matrix:

$$
M_{m n}=\sum_{\mathrm{ROI}}\left(\nabla f(\mathbf{x}) \cdot \boldsymbol{\psi}_{m}(\mathbf{x})\right)\left(\nabla f(\mathbf{x}) \cdot \boldsymbol{\psi}_{n}(\mathbf{x})\right),
$$

$\left\{\mathbf{b}^{i}\right\}$ the residual vector at iteration $i$ :

$$
b_{m}^{i}=\sum_{\mathrm{ROI}}\left(f(\mathbf{x})-\tilde{g}^{i}(\mathbf{x})\right)\left(\nabla f(\mathbf{x}) \cdot \boldsymbol{\psi}_{m}(\mathbf{x})\right)
$$

and $\tilde{g}^{i}(\mathbf{x})$ is equal to $g\left(\mathbf{x}+\mathbf{u}^{i}(\mathbf{x})\right)$ and referred to as the corrected deformed image by the current estimate $\mathbf{u}^{i}$ of the displacement field. In the present setting, the Hessian matrix $[\mathbf{M}]$ is calculated only once, whereas the residual vector $\left\{\mathbf{b}^{i}\right\}$ has to be updated for each new iteration step.

In addition, mechanical regularization based on the equilibrium gap method [11] is employed herein to further circumvent the ill-posedness of registration [12]. Such approach results in a reduction of uncertainty levels [12],[13]. If linear elasticity applies, and if the bulk nodes are free from external forces, the regularized FE-DIC consists of adding a penalty term:

$$
\Phi_{m}^{2}=\{\mathbf{u}\}^{\mathrm{T}}[\mathbf{K}]^{\mathrm{T}}[\mathbf{K}]\{\mathbf{u}\},
$$

where $[\mathbf{K}]$ is the stiffness matrix, while $\Phi_{m}^{2}$ is the sum of the squared norm of all equilibrium gaps at inner nodes only. Further, an additional regularization is applied for the loaded (Dirichlet) boundary nodes:

$$
\Phi_{b}^{2}=\{\mathbf{u}\}^{\mathrm{T}}[\mathbf{L}]^{\mathrm{T}}[\mathbf{L}]\{\mathbf{u}\}
$$

where $[\mathbf{L}]$ is the operator acting on the ROI boundary [14]. Thus, the regularized DIC consists of minimizing the total cost function $\Phi_{t}$ which comprises the correlation cost function $\left(\Phi_{c}^{2}\right)$, the equilibrium gap function $\left(\Phi_{m}^{2}\right)$ and the function of boundary fluctuations $\left(\Phi_{b}^{2}\right)$ : 
A. Vrgoč, Z. Tomičević

A. Zaplatić, F. Hild
Damage Analysis in Glass Fibre Reinforced Epoxy Resin via Digital Image Correlation

$$
\left(1+w_{m}+w_{b}\right) \Phi_{t}^{2}=\tilde{\Phi}_{c}^{2}+w_{m} \tilde{\Phi}_{m}^{2}+w_{b} \tilde{\Phi}_{b}^{2}
$$

where $w_{m}$ and $w_{b}$ are the weights defining the length scales associated with $\tilde{\Phi}_{m}^{2}$ and $\tilde{\Phi}_{b}^{2}$, while

$\tilde{\Phi}$ denotes the cost functions normalized with respect to those associated with the plane wave displacement field $\mathbf{v}(\mathbf{x})=\mathbf{v}_{0} \exp (i \mathbf{k} \cdot \mathbf{x})$, where $\mathbf{v}_{0}$ is the amplitude and $\mathbf{k}$ the wave vector. The normalized residuals read:

$$
\tilde{\Phi}_{c}^{2}=\frac{\Phi_{c}^{2}}{\{\mathbf{v}\}^{\mathrm{T}}[\mathbf{M}]\{\mathbf{v}\}}, \quad \tilde{\Phi}_{m}^{2}=\frac{\Phi_{m}^{2}}{\{\mathbf{v}\}^{\mathrm{T}}[\mathbf{K}]^{\mathrm{T}}[\mathbf{K}]\{\mathbf{v}\}}, \quad \tilde{\Phi}_{b}^{2}=\frac{\Phi_{b}^{2}}{\{\mathbf{v}\}^{\mathrm{T}}[\mathbf{L}]^{\mathrm{T}}[\mathbf{L}]\{\mathbf{v}\}},
$$

where $\{\mathbf{v}\}$ gathers all nodal degrees of freedom associated with the displacement field $\mathbf{v}$. The wavelength dependence of $\{\mathbf{v}\}^{\mathrm{T}}[\mathbf{K}]^{\mathrm{T}}[\mathbf{K}]\{\mathbf{v}\}$ and $\{\mathbf{v}\}^{\mathrm{T}}[\mathbf{L}]^{\mathrm{T}}[\mathbf{L}]\{\mathbf{v}\}$ is of fourth order, while $\{\mathbf{v}\}^{\mathrm{T}}[\mathbf{M}]\{\mathbf{v}\}$ is independent of the wavelength. Consequently, the weights $w_{m}$ and $w_{b}$ are chosen as:

$$
w_{m}=\left(2 \pi|\mathbf{k}| l_{m}\right)^{4}, \quad w_{b}=\left(2 \pi|\mathbf{k}| l_{b}\right)^{4},
$$

where $l_{m}$ and $l_{b}$ denote the regularization lengths for $\Phi_{m}^{2}$ and $\Phi_{b}^{2}$, respectively.

As previously mentioned, the DIC technique measures displacement fields, and thus enables the calculation of strain fields on the surface of tested specimens. Additionally, the correlation residual maps are available for each analysed image. These maps correspond to the grey level difference at the convergence of the correlation procedure (Equation (3)), thereby indicating the quality of registration. High values indicate local violations to the grey level conservation or incompleteness of kinematic bases, which make correlation residuals an ideal field for revealing the presence of cracks when displacement continuities are assumed to occur [15].

\subsection{Experimental protocol}

In order to analyse the global behaviour of the investigated composite, $5.8 \mathrm{~mm}$ thick dogbone specimens were subjected to monotonic tensile tests. The specimens were cut from a composite plate at $0^{\circ}, 45^{\circ}$ and $90^{\circ}$, as shown in Fig. 2(a).

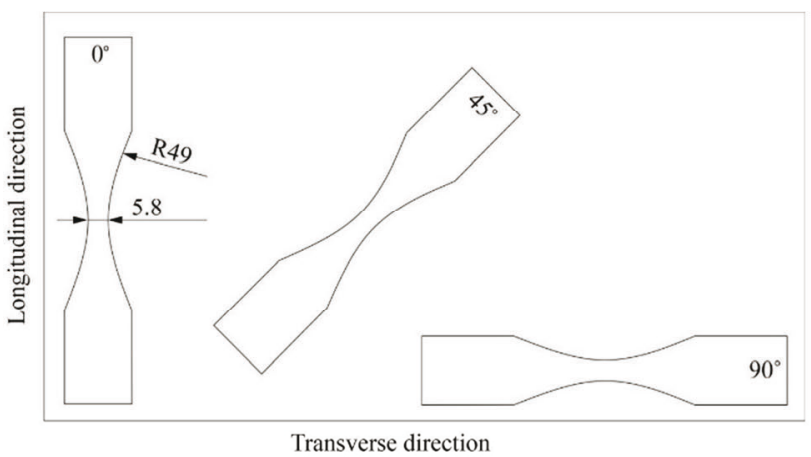

(a)

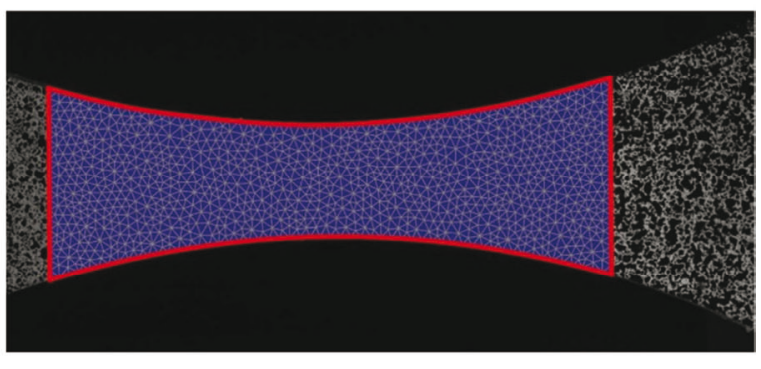

(b)

Fig. 2 Orientation of the specimens cut from the composite plate (a) and the reference image of the uniaxial tensile experiment used for the Digital Image Correlation analysis with 20-pixel triangular elements (b). The red contour depicts the Region Of Interest.

To ensure that the specimen breaks in the ligament area, the central part was thinned to a radius of $49 \mathrm{~mm}$, while the width at the narrowest section was equal to $5.8 \mathrm{~mm}$. 
Three uniaxial tensile tests were conducted in a displacement-controlled mode with a prescribed stroke rate of $2 \mathrm{~mm} / \mathrm{min}$. The tests were monitored with a single CCD camera, which resulted in 2D-DIC measurements. The image acquisition rate was equal to $3 \mathrm{fps}$. The three experiments were carried out consecutively without any changes to the camera and lighting settings. Furthermore, in order to measure displacement fields, a fine-grained random black and white speckle pattern was applied to the surface of the specimens. The ROI considered herein is shown in Fig. 2(b), while the hardware parameters of the optical setup for DIC measurements are summarized in Table 1.

Table 1 Digital Image Correlation hardware parameters.

\begin{tabular}{|c|c|}
\hline Camera & CCD Dalsa \\
Definition & $2358 \times 1728$ pixels \\
Grey levels amplitude & $8 \mathrm{bits}$ \\
Lens & Titanar $50 \mathrm{~mm}$ \\
Aperture & $f / 2.8$ \\
Field of view & $38 \times 28 \mathrm{~mm}^{2}$ \\
Image scale & $16 \mu \mathrm{m} / \mathrm{pixel}$ \\
Stand-off distance & $120 \mathrm{~mm}$ \\
Image acquisition rate & $3 \mathrm{fps}$ \\
Exposure time & $26 \mathrm{~ms}$ \\
Patterning technique & Sprayed black paint \\
\hline
\end{tabular}

The images acquired during each test were analysed via a regularized FE-based DIC within the Correli 3.0 framework [16]. Since the correlation code estimates the nodal displacements associated with the finite element discretization, a 2D mesh corresponding to the specimen geometry and consisting of three-node triangular (T3) elements was considered herein (Fig. 2(b)). The T3 elements were selected to properly describe the boundaries of each specimen. The mesh topology was identical for each specimen. However, the meshes slightly differed in shape owing to variations in specimen alignment. The average element length, calculated as the square root of the mean element area, was equal to $20 \mathrm{px}$ for each specimen. The other parameters employed in the correlation analyses are summarized in Table 2.

Table 2 Digital Image Correlation analysis parameters.

\begin{tabular}{|c|c|}
\hline DIC software & Correli 3.0 [16] \\
Image filtering & None \\
Element length & 20 pixels \\
Shape functions & Linear (T3) \\
Mesh & See Fig. 2(b) \\
Matching criterion & Regularized sum of squared differences \\
Regularization length & 10 pixels \\
\hline
\end{tabular}

Before performing DIC measurements, the displacement and strain uncertainties were quantified by acquiring 50 images in the reference configuration. Their levels obtained from each test are shown in Table 3. For all three cases, the uncertainties are identical for the maximum eigen strain, and the displacement uncertainties are in the cpx range. Even though finer FE meshes better capture kinematic fields (especially in the presence of localized phenomena), they are more corrupted by acquisition noise (i.e. the lower the element size, the higher measurement uncertainties). This observation leads to a compromise between measurement uncertainty and spatial resolution [10]. Hence, small scale displacement 
resolutions are hardly accessible without introducing more assumptions (e.g. mechanical regularization) in the correlation procedure. When the regularization length is greater than the element size, the former then controls the spatial resolution. Otherwise, the element size is proportional to the spatial resolution.

Table 3 Standard displacement and strain uncertainties.

\begin{tabular}{|c|cc|}
\hline Displacement noise floor $0^{\circ}(x y)$ & $0.012 \mathrm{px}$ & $0.012 \mathrm{px}$ \\
Displacement noise floor $45^{\circ}(x y)$ & $0.016 \mathrm{px}$ & $0.013 \mathrm{px}$ \\
Displacement noise floor $90^{\circ}(x y)$ & $0.017 \mathrm{px}$ & $0.011 \mathrm{px}$ \\
Strain noise $\varepsilon_{1}$ floor $0^{\circ}, 45^{\circ}$ and $90^{\circ}$ & \multicolumn{2}{|c|}{$8 \times 10^{-5}$} \\
\hline
\end{tabular}

\section{Results}

The main objective of the research reported herein was to monitor damage initiation and growth on the surface of glass fibre/epoxy resin composite via DIC. An FE-based DIC was carried out to measure displacement fields and calculate strain fields. In addition, the grey level residual maps were investigated to detect discontinuities and monitor damage growth on the specimen surfaces.

\subsection{Global material behaviour}

The global material response in the uniaxial tensile tests was obtained via DIC gauges positioned over the ROI, as shown in Fig. 3(a). The uniaxial stress was determined from the load data and the initial area of the ligament. From the reported stress-strain curves, Young's modulus values of $6.8 \mathrm{GPa}, 5.4 \mathrm{GPa}$, and $5.5 \mathrm{GPa}$ were identified for the $0^{\circ}, 45^{\circ}$, and $90^{\circ}$ specimens, respectively. Fig. 3(a) shows good agreement between stress-strain curves in the linear elastic regime for the $45^{\circ}$ and $90^{\circ}$ specimens, but not for the $0^{\circ}$ specimen. This difference arises from the specimen machining conditions as well as from the presence of voids (Fig. 1) within the specimens. A detailed microstructural analysis was performed on the X-Ray Computed Tomography (CT) scans to explain the differences in the obtained results (Fig. 3(b)). The reconstructed mesostructure reveals a random glass fibre architecture and provides some information about the distribution of voids within the specimen.

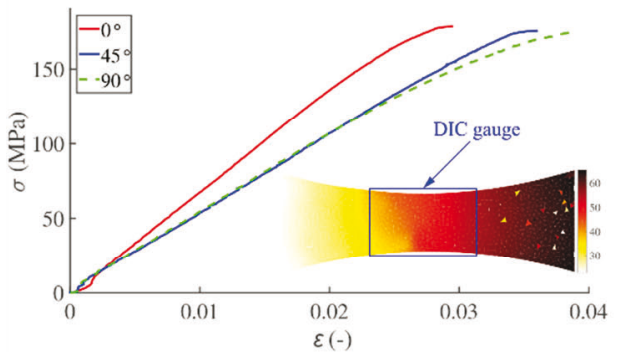

(a)



(b)

Fig. 3 Stress-strain curves of all three tensile tests (a) and the mesostructure of the chosen ROI (b). The reconstructed volume reveals the random fibre orientation and the presence of voids (i.e. air bubbles).

\subsection{Correlation results for the $0^{\circ}$ specimen}

A total of 313 images were analysed for the $0^{\circ}$ specimen. The first 50 images were obtained in the unloaded configuration for uncertainty quantification purposes (Table 3). Table 4 lists both the maximum eigen strain and stress data calculated for different images during the prescribed load history. In the present case, the residual maps and maximum eigen strain fields for the images listed in Table 4 are reported to detect damage initiation and growth. Fig. 4 shows the corresponding stress-strain curve, in which the studied pictures are 
shown, and the change in the RMS residual with respect to the image number. Up to Image 50 , the global residual is constant since no loading was prescribed. As the loading increases monotonically, a gradual increase in the RMS residual is observed up to Image 180, which corresponds to the linear elastic material response in Fig. 4. Above $112 \mathrm{MPa}$ (Image 180) material nonlinearity is observed.

Table 4 Stress and maximum eigen strain values of the $0^{\circ}$ specimen calculated for different load levels.

\begin{tabular}{|c|c|c|}
\hline Image number & Stress $(\mathrm{MPa})$ & $\varepsilon_{1}$ \\
\hline 50 & - & - \\
90 & 30 & 0.004 \\
144 & 76 & 0.012 \\
180 & 118 & 0.016 \\
245 & 160 & 0.024 \\
299 & 184 & 0.037 \\
\hline
\end{tabular}

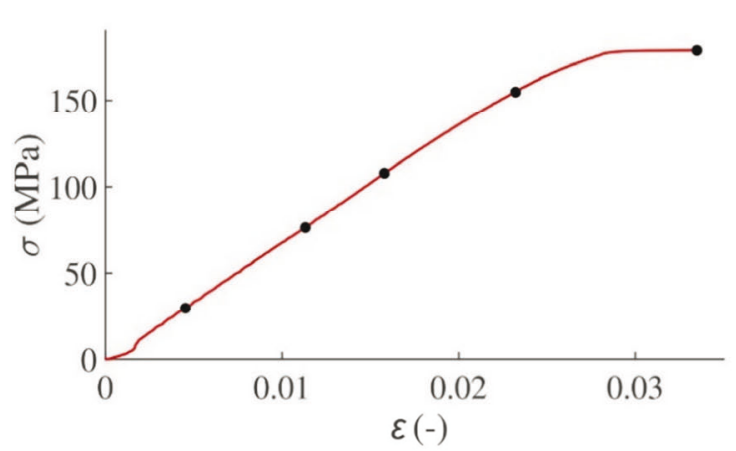

(a)

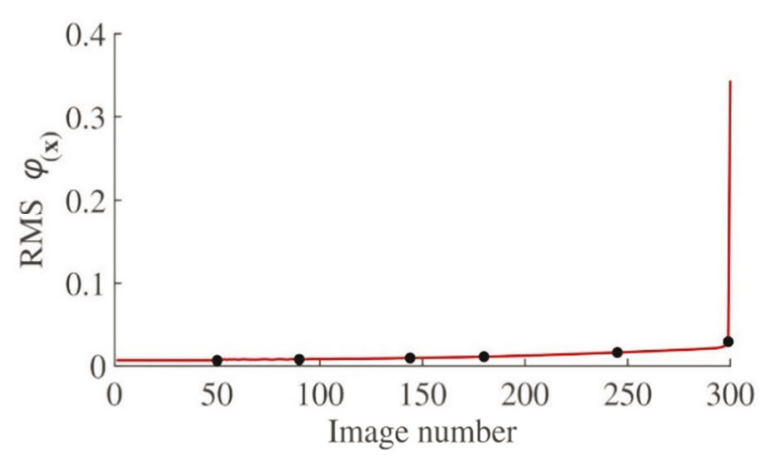

(b)

Fig. 4 A stress-strain curve obtained from the monotonic uniaxial tensile test (a), and the change in the normalized RMS residuals as a function of image number (b) extracted from the correlation analysis of the $0^{\circ}$ specimen. The solid black circles depict the images for which the correlation residual maps are shown to detect discontinuities.

Figure 5 shows the grey level residual maps and the corresponding maximum eigen strain fields for different load levels in the linear elastic regime of the material response. The dynamic range of the grey level residuals is selected so that an increase in fluctuations is observed. Fig. 5(a)-(b) show the initial residual map and the maximum eigen strain field calculated in the unloaded regime. Very low residuals are homogeneously distributed over the ROI, thus indicating that the registration was successful. These low initial residuals were induced by the camera sensor noise during image acquisition. Further, the strain fluctuations of small magnitude are due to acquisition noise. As the load levels become higher, the correlation residuals gradually increase over the entire ROI (Fig. 5(c)). The first detected discontinuities correspond to Image 144, i.e. four distinct regions of elevated residuals are observed at the upper and the lower edge of the specimen ligament (Fig. 5(c)). Such localized phenomena are also visible in the maximum eigen strain field (Fig. 5(d)). Thus, these strain concentrations on the edges of the specimen ligament show the impact of damage induced by the specimen machining.

With a gradual increase in load levels, localized phenomena become dominant and the presence of a macrocrack within the narrowest gauge section is visible in both fields (Fig. 6(a)-(b)). Consequently, the damage growth induces a nonlinear material response (Fig. 4(a)). Additional regions of elevated grey level residuals and maximum eigen strains are observed on the edges of the specimen ligament (marked with red circles in Fig. 6(c)-(d)). 
Further, as shown in Fig. 6(e)-(f), which correspond to the last image acquired prior to the specimen failure, strain localization phenomena become more apparent, while the correlation residual map shows discontinuities within the same regions. It is worth noting that the damage process concentrates around the dominant macrocrack even though multiple areas with elevated correlation residuals and maximum eigen strains are detected as well. Failure occurs within the narrowest gauge section of the dog bone specimen (which was expected from its design).

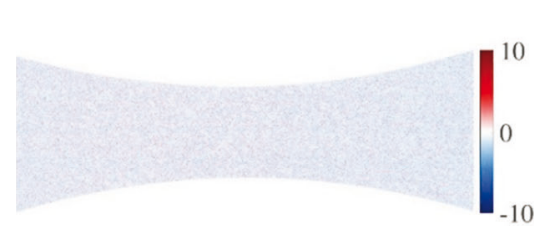

(a) $\varphi_{\mathrm{c}}$ at Image 50

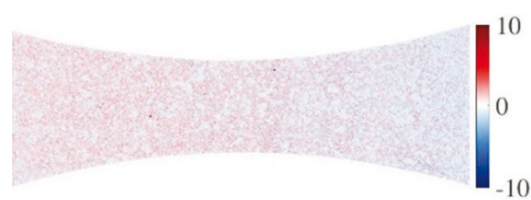

(c) $\varphi_{\mathrm{c}}$ at Image 90



(e) $\varphi_{\mathrm{c}}$ at Image 144

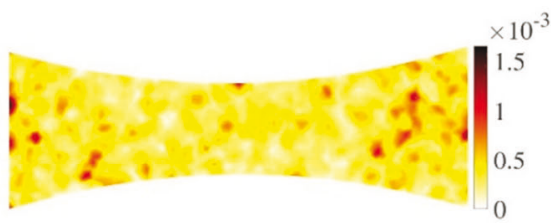

(b) $\varepsilon_{1}$ at Image 50

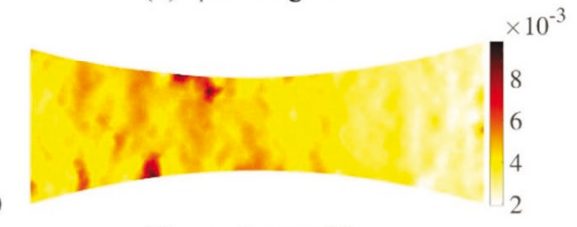

(d) $\varepsilon_{1}$ at Image 90



(f) $\varepsilon_{1}$ at Image 144

Fig. 5 Grey level residual maps (left) and maximum eigen strain fields (right) for three different loading steps in the linear elastic regime of the $0^{\circ}$ tensile test. The black circles depict the first detected discontinuities (i.e. zones in which the eigen strains increase with the prescribed load).

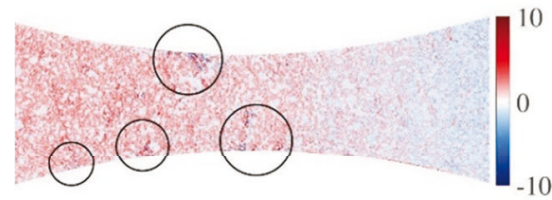

(a) $\varphi_{\mathrm{c}}$ at Image 180

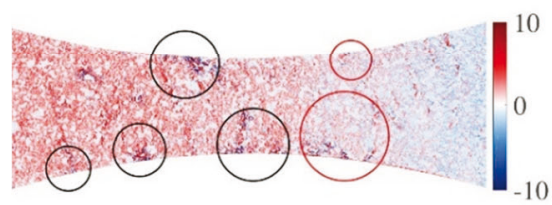

(c) $\varphi_{\mathrm{c}}$ at Image 245



(e) $\varphi_{\mathrm{c}}$ at Image 299

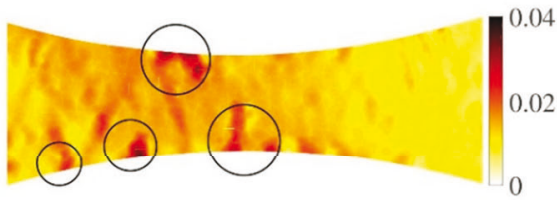

(b) $\varepsilon_{1}$ at Image 180

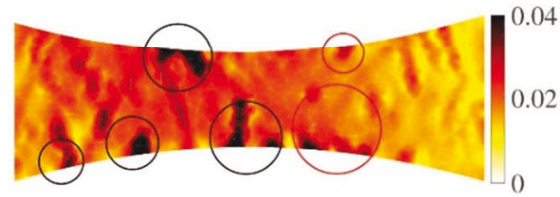

(d) $\varepsilon_{1}$ at Image 245

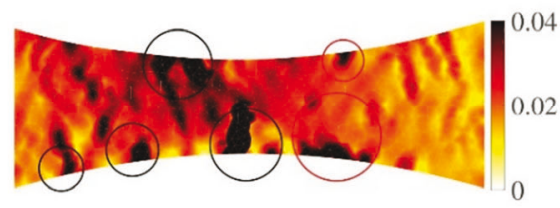

(f) $\varepsilon_{1}$ at Image 299

Fig. 6 Grey level residual maps (left) and maximum eigen strain fields (right) for different loading steps in the nonlinear regime of the $0^{\circ}$ tensile test. The black circles depict the first detected discontinuities, while the red circles correspond to additional areas of higher correlation residuals.

Fig. 7(a) shows the displacement field measured in the longitudinal direction prior to failure. The discontinuity in the displacement field is large and corresponds to the previously observed dominant macrocrack revealed through both the grey level residuals and maximum 
eigen strain field (Fig. 7(b)). The observed macrocrack path (marked with the black dashed line) is not straight, thereby indicating the influence of the random and heterogeneous mesostructure.

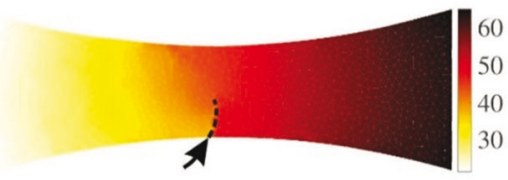

(a) $U_{\mathrm{x}}(\mathrm{px})$

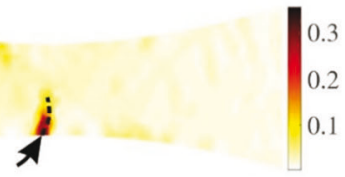

(b) $\varepsilon_{1}$

Fig. 7 Displacement field of the $0^{\circ}$ specimen in the loading direction (i.e. $x$ axis) expressed in pixels (a) and maximum eigen strain field (b) for Image 299. The black dashed line depicts the macrocrack path and the black arrow shows the initiation location.

\subsection{Correlation results for the $45^{\circ}$ and $90^{\circ}$ specimens}

A total of 314 images were analysed to detect damage on the $45^{\circ}$ specimen surface, while 306 images were analysed for the $90^{\circ}$ specimen. In both cases, the first 50 images were acquired in the unloaded stage to assess the measurement uncertainties (Table 3). Fig. 8(a)-(b) show the initial residual map and the maximum eigen strain field of the $45^{\circ}$ specimen corresponding to Image 50. The correlation residuals are homogeneously distributed over the ROI, while their values remain low, again indicating the influence of the optical setup. As the load levels become higher, the correlation residuals gradually increase over the entire ROI (Fig. 8(c)). The first detected discontinuities correspond to Image 154 captured in the linear elastic regime (Fig. 8(c)), i.e. three distinct regions of elevated residuals are observed at the upper and the lower edge of the specimen ligament. Such localized phenomena are also visible in the maximum eigen strain field (Fig. 8(d)). These strain concentrations on the edges of the specimen ligament show the impact of the damage induced by the specimen machining. Further, as shown in the images acquired prior to the specimen failure (Fig. 8(e)-(f)), strain localization phenomena become even clearer and correspond to the regions of elevated correlation residuals.

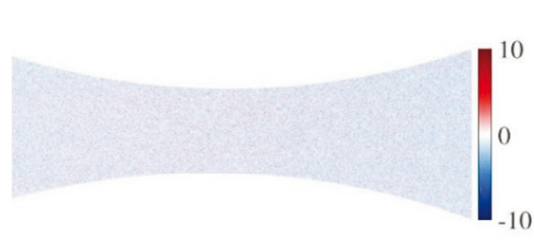

(a) $\varphi_{\mathrm{c}}$ at Image 50

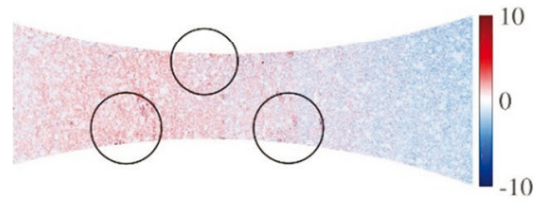

(c) $\varphi_{\mathrm{c}}$ at Image 154

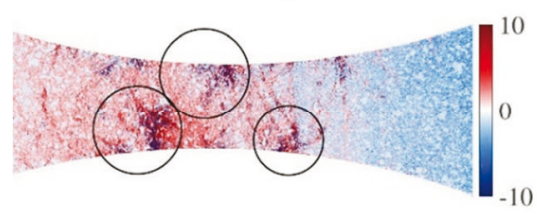

(e) $\varphi_{\mathrm{c}}$ at Image 307



(b) $\varepsilon_{1}$ at Image 50

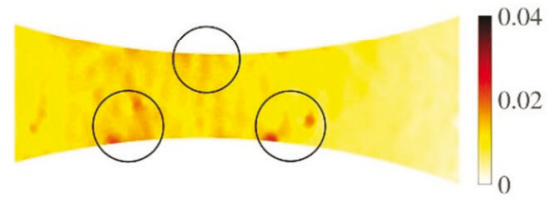

(d) $\varepsilon_{1}$ at Image 154

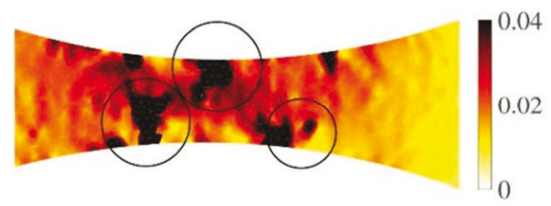

(f) $\varepsilon_{1}$ at Image 307

Fig. 8 Grey level residual maps (left) and the maximum eigen strain fields (right) for different loading steps of the $45^{\circ}$ tensile test. The black circles depict the first detected discontinuities.

Fig. 9(a)-(b) show the initial correlation residual map of the $90^{\circ}$ specimen for Image 50 , as well as the maximum eigen strain field. The correlation residuals are homogeneously 
distributed over the ROI, while the small fluctuations in the maximum eigen strain are due to measurement uncertainties. The increase in load level results in a gradual increase in correlation residuals over the entire ROI (Fig. 9(c)). The first detected discontinuities correspond to Image 160 captured in the linear elastic regime (Fig. 9(c)), i.e. four distinct regions of elevated residuals are observed at the upper and the lower edge of the specimen ligament. Such localized events are also visible in the maximum eigen strain field (Fig. 9(d)). Higher correlation residuals are even more evident in Fig. 9(e), which is the last image acquired prior to specimen failure. Strain localization phenomena are also visible in Fig. 9(f). The damage process concentrates around the dominant macrocrack, although multiple regions with elevated correlation residuals are detected as well.

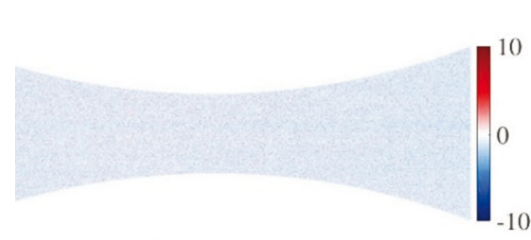

(a) $\varphi_{\mathrm{c}}$ at Image 50

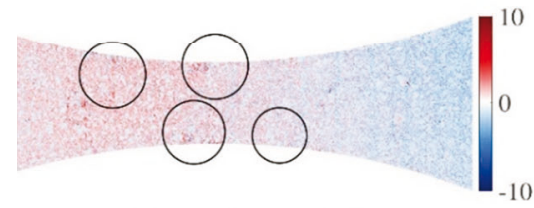

(c) $\varphi_{\mathrm{c}}$ at Image 160

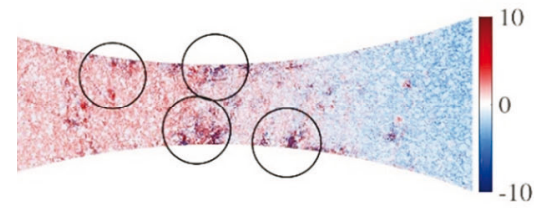

(e) $\varphi_{\mathrm{c}}$ at Image 294

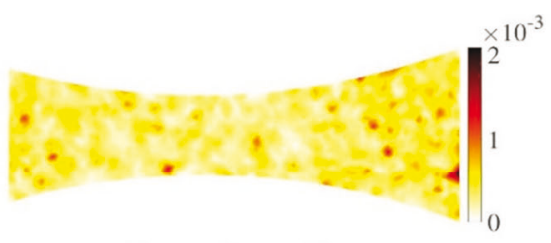

(b) $\varepsilon_{1}$ at Image 50

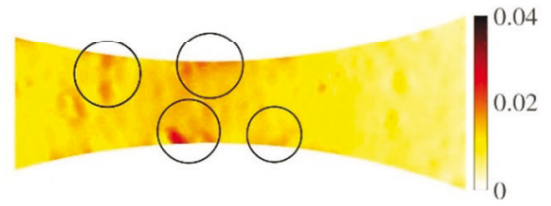

(d) $\varepsilon_{1}$ at Image 160

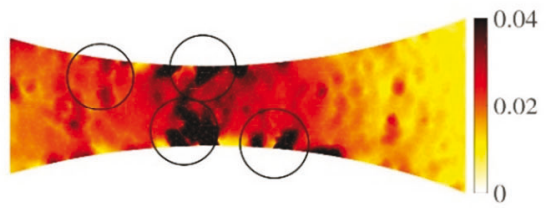

(f) $\varepsilon_{1}$ at Image 294

Fig. 9 Grey level residual maps (left) and the maximum eigen strain fields (right) for different loading steps of the $90^{\circ}$ tensile test. The black circles depict the first detected discontinuities.

Fig. 10 shows the displacement fields measured in the longitudinal direction prior to the failure of the $45^{\circ}$ and $90^{\circ}$ specimens.

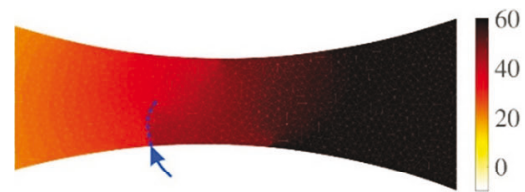

(a) $U_{x}(\mathrm{px})$ of $45^{\circ}$ sample

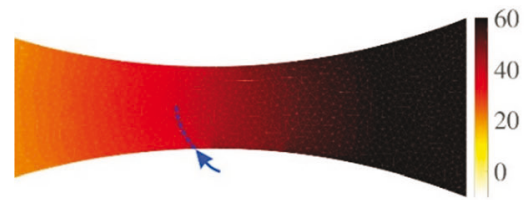

(c) $U_{x}(\mathrm{px})$ of $90^{\circ}$ sample

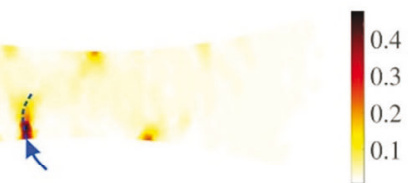

(b) $\varepsilon_{1}$ of $45^{\circ}$ sample



(d) $\varepsilon_{1}$ of $90^{\circ}$ sample

Fig. 10 Displacement field in the loading direction (left) and the maximum eigen strain field (right) of the $45^{\circ}$ and $90^{\circ}$ specimens corresponding to the last images acquired prior to specimen failure. The displacements are expressed in pixels; the blue dashed lines depict the macrocrack path and the blue arrows show the microcrack initiation locations.

The discontinuities in the measured displacement fields are large and correspond to the previously observed discontinuities revealed through the grey level residuals and the maximum eigen strain fields. In both cases, the dominant macrocrack path is not straight, which indicates the influence of underlying heterogeneities. 


\section{Conclusion}

Monotonic tensile tests were carried out on a glass fibre mat/epoxy resin composite. Two-dimensional digital image correlation (2D-DIC) was employed to measure displacements and calculate strain fields on the surface of the investigated material. First, the global material response in the uniaxial tensile tests was obtained via DIC gauges positioned over the region of interest, and Young's modulus values for all three specimens were identified. Good agreement between stress-strain curves in the linear elastic regime for the $45^{\circ}$ and $90^{\circ}$ specimens was observed (Young's modulus equal to $5.5 \mathrm{GPa}$ and $5.4 \mathrm{GPa}$, respectively). However, Young's modulus for the $0^{\circ}$ specimen was $6.8 \mathrm{GPa}$. It is worth noting that the observed fluctuations in mechanical response originated from the random fibre architecture, as well as from the specimen machining and the presence of mesodefects (i.e. air bubbles) resulting from the manufacturing process. A microstructural analysis was conducted by acquiring a $3 \mathrm{D}$ scan of the chosen ROI. The 3D reconstructed volume revealed the underlying fibre mat architecture, as well as the presence of voids.

The analysis of DIC residuals and maximum eigen strain fields revealed damage initiation and growth on the surface of investigated specimens. For all three specimens, the initial correlation residuals corresponding to the unloaded state were homogeneously distributed over the ROI and their levels remained low, thereby indicating the influence of the optical experimental setup (i.e. lighting, acquisition parameters, and camera sensor noise). As the load levels became higher, those residuals gradually increased over the entire ROI. The first detected discontinuities for all three specimens were observed at the upper and the lower edge of the specimen ligament in the linear elastic regime, thus indicating the impact of the damage induced by the specimen machining (since the observed localization phenomena did not affect the global material response, i.e. the nonlinearity was still very low). Localizations observed on the correlation residuals were also visible on the maximum eigen strain fields. With higher load levels, localized phenomena became dominant and the presence of a macrocrack and its path were revealed. The observed macrocrack path for all three specimens was not straight due to the underlying heterogeneities. Because of the random specimen mesostructure and the presence of defects induced by the manufacturing and machining, the specimens broke at different stress and elongation levels.

The results obtained in this study indicate that the methodology employed herein (regularized FE-DIC) can efficiently detect damage and macrocracks on the surface of composite materials. However, DIC measurements do not provide a comprehensive insight into the behaviour of a fibre-reinforced polymer and the underlying damage mechanisms in the material bulk. This type of analysis can be achieved via in-situ X-ray computed tomography (CT) [17]. The reconstructed volumes can be registered via Digital Volume Correlation (DVC) to measure 3D displacement fields [18],[19]. Such approach would enable the identification of damage mechanisms at the microscale and in the bulk, as well as the calibration of constitutive parameters.

\section{Acknowledgements}

This research was conducted within the FULLINSPECT project supported by the Croatian Science Foundation (UIP-2019-04-5460 Grant).

\section{REFERENCES}

[1] Brighenti, R., Carpinteri, A., Scorza, D. Mechanics of interface debonding in fiber-reinforced materials, Journal of Composite Materials 2016, 50, 2699-2718. https: \doi.org \10.1177/0021998315612537

[2] Shi, Y., Swait, T., Soutis, C. Modelling damage evolution in composite laminates subjected to low velocity impact, Composite Structures 2012, 94, 2902-2913. https:Idoi.org|0.1016/J.compstruct.2012.03.039 
[3] Schwab, M., Todt, M., Pettermann, H. A multiscale approach for modelling impact on woven composites under consideration of the fabric topology, Journal of Composite Materials 2018, 52, 2859-2874. https: \doi.org $\backslash 10.1177 / 0021998318755865$

[4] Sutton M.A., Orteu JJ., Schreier H. (2009) Digital Image Correlation (DIC). In: Image Correlation for Shape, Motion and Deformation Measurements. Springer, Boston, MA. https://doi.org/10.1007/978-0-387-78747-3_5

[5] Bornert, M., Hild, F., Orteu, J.-J. and Roux, S. (2013). Digital Image Correlation. In Full-Field Measurements and Identification in Solid Mechanics (eds M. Grédiac, F. Hild and A. Pineau). https://doi.org/10.1002/9781118578469.ch6

[6] Sutton, M. Computer Vision-Based, Noncontacting Deformation Measurements in Mechanics: A Generational Transformation, Applied Mechanics Reviews 2013, 65, 050802. https:\doi.org $\backslash 10.1115 / 1.4024984$

[7] Gras, R., Leclerc, H., Roux, S., Otin, S., Schneider, J., Périé, J. Identification of the Out-of-Plane Shear Modulus of a 3D Woven Composite, Experimental Mechanics 2013, 53, 719-730. https:〈doi.org \10.1007/S11340-012-9683-4

[8] Tomičević, Z., Bouterf, A., Surma, R., Hild, F. Damage observation in glass fiber reinforced composites via $\mu$-tomography, Materials Today: Proceedings 2019, 12, 185-91. https:〈doi.org\10.1016/J.MATPR.2019.03.093

[9] Montesano, J., Selezneva, M., Lévesque, M., Fawaz, Z. Modeling fatigue damage evolution in polymer matrix composite structures and validation using in-situ digital image correlation, Composite Structures 2015, 125, 354-361. https:\doi.org \10.1016/J.compstruct.2015.02.035

[10] Hild, F., Roux, S. Comparison of Local and Global Approaches to Digital Image Correlation, Experimental Mechanics 2012, 52, 1503-1519. https: \doi.org \10.1007/S11340-012-9603-7

[11] Claire, D., Hild, F., Roux, S. A finite element formulation to identify damage fields: the equilibrium gap method, International Journal for Numerical Methods in Engineering 2004, 61, 189-208. https:\doi.org \10.1002/NME.1057

[12] Tomičević, Z., Hild, F., Roux, S. Mechanics-aided digital image correlation, The Journal of Strain Analysis for Engineering Design 2013, 48, 330-343. https: \doi.org \10.1177/0309324713482457

[13] Naylor, R., Hild, F., Fagiano, C., Hirsekorn, M., Renollet, Y., Tranquart, B., Baranger, E. Mechanically regularized FE DIC for heterogeneous materials, Experimental Mechanics 2019, 59, 1159-1170. https: \doi.org $\backslash 10.1007 / \mathrm{S} 11340-019-00529-9$

[14] Mendoza, A., Neggers, J., Hild, F., Roux, S. Complete mechanical regularization applied to digital image and volume correlation, Computer Methods in Applied Mechanics and Engineering 2019, 355, 27-43. https: \doi.org $\backslash 10.1016 / J . C M A .2019 .06 .005$

[15] Hild, F., Bouterf, A., Roux, S. Damage measurements via DIC, International Journal of Fracture 2015, 191, 77-105. https: \doi.org \10.1007/s10704-015-0004-7

[16] Leclerc, H., Neggers, J., Mathieu, F., Hild, F., Roux, S. 2015, Correli 3.0, Agence pour la Protection des Programmes, IDDN.FR.001.520008.000.S.P.2015.0.0.0.31500

[17] Maire, E., Withers, P. Quantitative X-ray tomography, International Materials Reviews 2014, 59, 1-43. https: \doi.org $\backslash 10.1179 / 1743280413$ Y.0000000023

[18] Bay, B. Methods and applications of digital volume correlation. The Journal of Strain Analysis for Engineering Design 2008, 43, 745-760. https: \doi.org \10.1243/03093247JSA436

[19] Buljac, A., Jailin, C., Mendoza, A., Neggers, J., Taillandier-Thomas, T., Bouterf, A., Smaniotto, B., Hild, F., Roux, S. Digital Volume Correlation: Review of Progress and Challenges, Experimental Mechanics 2018, 58, 661-708. https: \doi.org \10.1007/S11340-018-0390-7

Submitted: $\quad 06.11 .2020$

Accepted: $\quad 18.02 .2021$
Ana Vrgoč

Zvonimir Tomičević

Andrija Zaplatić

Faculty of Mechanical Engineering and

Naval Architecture, University of Zagreb François Hild

Université Paris-Saclay, ENS ParisSaclay, CNRS, LMT - Laboratoire de mécanique et technologie, 91190 Gif-surYvette, France 